\title{
Tubercular Esophagocutaneous Fistula
}

\author{
Ranendra Hajong • Noor Topno • Arup Jyoti Baruah • \\ Rubul Das
}

Received: 4 August 2010 / Accepted: 20 February 2011 / Published online: 12 May 2011

(C) Association of Surgeons of India 2011

\begin{abstract}
Tubercular esophagocutaneous fistula is a rare entity with only about four cases reported so far. We report here a case in a young female who has a very long tract but responded well to antitubercular treatment.
\end{abstract}

Keywords Tuberculosis $\cdot$ Fistula $\cdot$ Esophagocutaneous

\section{Introduction}

Tuberculosis of the esophagus is exceedingly rare, occurring in less than $0.2 \%$ of all tuberculosis patients [1-4]. It usually results from a spread of an adjacent focus, such as the lung or mediastinal nodes, spine, larynx or pharynx and tends to involve the proximal rather than the distal esophagus [1-6].The most common presenting symptoms, in addition to the systemic symptoms of tuberculosis, are dysphagia, epigastric pain and odynophagia [7] Esophagocutaneous fistula is a very rare complication of tuberculosis [7].

\section{Case Report}

A 24 year old female of average built presented with a complaint of a non-healing ulcer on the back (Fig. 1) for the

R. Hajong $(\bowtie) \cdot$ N. Topno $\cdot$ A. J. Baruah $\cdot$ R. Das

Department of General Surgery, NEIGRIHMS,

Shillong 793018, India

e-mail: ranenhajong@gmail.com last 4 months. She had tried some local medications before approaching the hospital. The patient gave a history of food particles coming out occasionally from the opening, evening rise of temperature and recent loss of weight. Her HIV status was normal. Upper gastrointestinal endoscopy showed an internal opening about $25 \mathrm{~cm}$ from the incisor tooth (Fig. 2) Biopsy showed caseating epitheloid granulomas with AFB positivity. Chest x-ray showed complete collapse of the right lung with compensatory left lung hypertrophy (Fig. 3). The fistula tract was cannulated and fistulogram obtained (Fig. 4). The patient was subsequently started on antitubercular treatment according to the DOTS regime and on follow-up after 4 months, the discharge from the fistula has stopped and there was no pleural effusion. The patient also gained about $10 \mathrm{~kg}$ of weight.

\section{Discussion}

Esophageal tuberculosis can be primary or secondary. Usually it is secondary to tuberculous caseation involving the neighboring structures [8]. In the setting of an esophageal stricture or ulceration, swallowed infected sputum can give rise to primary mucosal involvement of the esophagus [8]. In the case reported by Yokoyama et al. [9], the fistula had resulted from a lung abscess and mediastinal lymphadenopathy and the patient required surgical repair of the fistula. A similar case was described by Xavier et al. [10] in an elderly patient without lung abscess but in whom the mediastinal lymph nodes were 


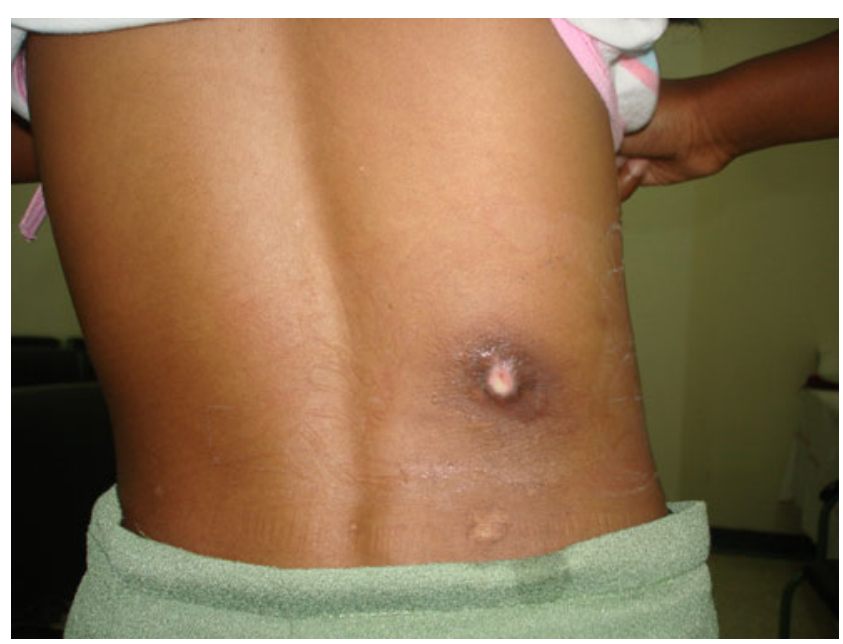

Fig. 1 Showing external opening

the likely source. The patient died due to drug-induced hepato-toxicity. In the case reported by David et al. [11], long-standing pulmonary tuberculosis with mediastinal lymphadenopathy led to fistula formation. In our patient the likely source could be pulmonary tuberculosis on the right side with mediastinal lymphadenopathy leading to the fistula. CT scan of the thorax could not be done as the patient could not afford the same.

The differential diagnosis of the fistulous tracts of the esophagus includes malignancy, Crohn's disease, radiation injury and trauma. The diagnosis can be confirmed by demonstration of the fistulous tract with radiocontrast studies. Computed tomographic scanning with oral and

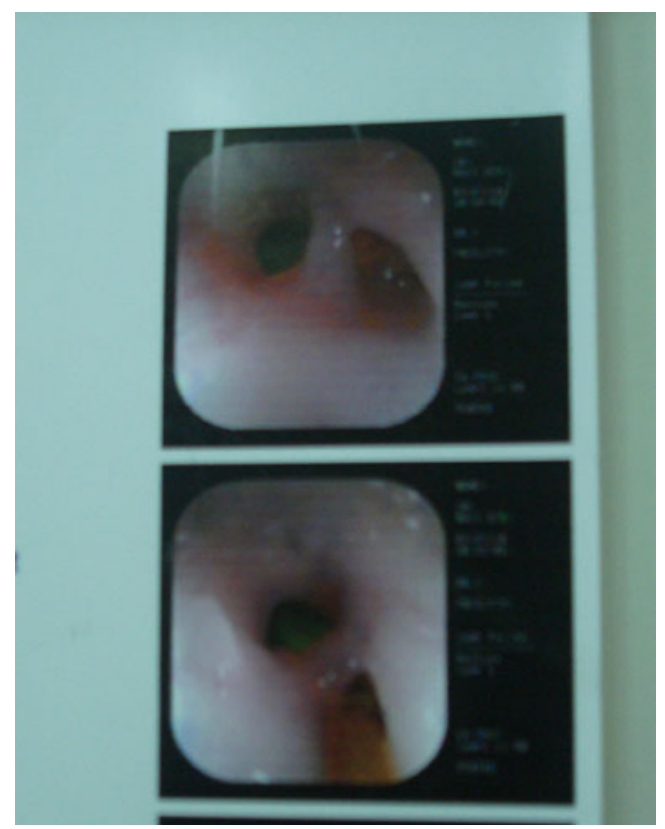

Fig. 2 Showing esophageal end of the fistula

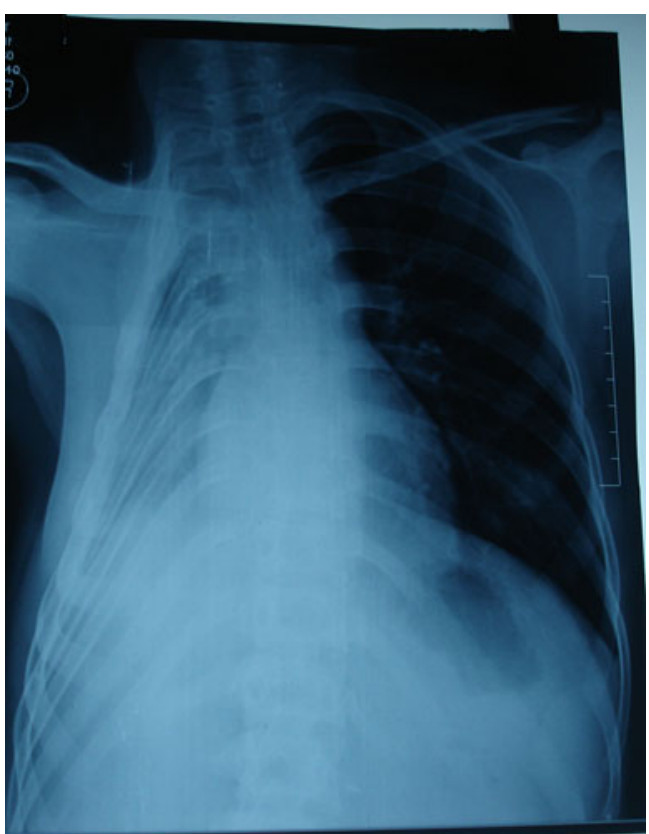

Fig. 3 Showing completely collapsed ling on the right side

intravenous contrast is useful to delineate fistulous tracts and mediastinal adenopathy [12]. Pathologic and microbiologic confirmation can be obtained by biopsy and culture of sinus tract endoscopically or percutaneously. Esophageal tuberculosis is reported to respond well to medical treatment in 4-8 weeks [12-14] but one of the cases reported by Yokoyama et al. required surgery as definitive therapy [9]. Our patient was started on ATT and is doing well.

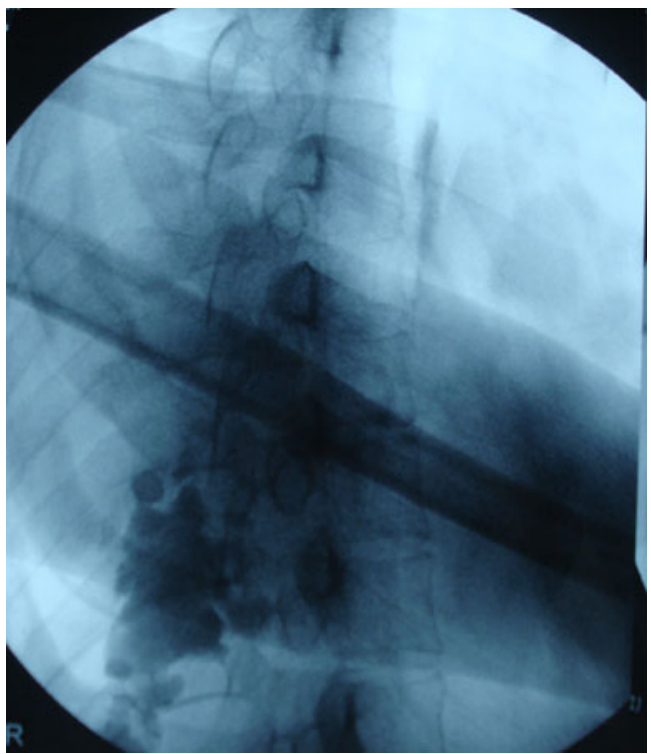

Fig. 4 Showing fistulogram 


\section{References}

1. Sathiyasekaran M, Shivbalan S (2004) Esophageal tuberculosis. Indian J Pediatr 71:457-458

2. Shah S (2002) Esophageal tuberculosis: is it so rare? Report of 12 cases and review of the literature. Indian J Gastroenterol 21:170

3. Jain SK, Jain S, Jain M, Yaduvanshi A (2002) Esophageal tuberculosis: is it so rare? Report of 12 cases and review of the literature. Am J Gastroenterol 97:287-291

4. Sharma MP, Bhatia V (2004) Abdominal tuberculosis. Indian J Med Res 120:305-315

5. Bhatnagar MS, Nanivadekar SA, Sawant P, Rathi PM, Upadhaya AP (1996) Asymptomatic spinal tuberculosis presenting as esophageal stricture. Indian J Gastroenterol 15:72-73

6. Devarbhavi HC, Alvares JF, Radhikadevi M (2003) Esophageal tuberculosis associated with esophagotracheal or esophagomediastinal fistula: report of 10 cases. Indian J Surg 57:588-592

7. Vinodh BN, Sharma SK, Smith-Rohrberg D, Seith A (2006) Tubercular esophagocutaneous fistula. Indian J Chest Dis Allied Sci 48:209-211
8. Gordon AH, Marshal JB (1990) Esophageal tuberculosisdefinite diagnosis by endoscopy. Am J Gastroenterol 85:174 177

9. Yokoyama I, Ichihashi H, Kawahara K (1984) Esophagopleurocutaneous fistula. A case report. Jpn J Surg 14:139142

10. Xavier S, Kochhar R, Nagi B, Singh K (1996) Tuberculous esophagocutaneous fistula. J Clin Gastroenterol 23:118120

11. David Chausse J, Dehais J, Bullier R et al (1977) Pott's dorsal disease, mediastinal suppuration, esophageal tuberculosis with cutaneous fistulas and cold thoracic abscesses. Rev Rhum Mal Osteoartic 44:193-197

12. Im JG, Kim JH, Han MC et al (1990) Computerised tomography of esophagomediastinal fistula in tuberculous mediastinal lymphadenitis. J Comput Assist Tomogr 14:89-92

13. Eng J, Sabanathan S (1991) Tuberculosis of the esophagus. Dig Dis Sci 36:536-540

14. Mokoena T, Sharma OM, Ngakane H et al (1992) Esophageal tuberculosis: a review of eleven cases. Postgrad Med J 68:110 115 